Acta Crystallographica Section A

Foundations of Crystallography

ISSN 0108-7673

Received 9 October 2007

Accepted 2 November 2007

(C) 2008 International Union of Crystallography

Printed in Singapore - all rights reserved

\section{Report on a project on three-dimensional imaging of the biological cell by single-particle $\mathrm{X}$-ray diffraction}

\author{
D. Sayre
}

Department of Physics and Astronomy, Stony Brook University, Stony Brook, NY 11794-3800, USA. Correspondence e-mail: sayre@xray1.physics.sunysb.edu

Single-particle X-ray diffraction is an extension of X-ray crystallography which allows the specimen to be any small solid-state bounded object; in Shapiro et al. [Proc. Natl Acad. Sci. USA (2005), 102, 15343-15346] and Thibault et al. [Acta Cryst. (2006), A62, 248-261], the reader can find descriptions of a recent StonyBrook/Berkeley/Cornell two-dimensional imaging of a yeast cell by this technique. Our present work is aimed at extending the technique to the threedimensional imaging of a cell. However, the usual method of doing that, namely rotating the specimen into many orientations in the X-ray beam, has not as yet given sufficiently good three-dimensional diffraction data to allow the work to go forward, the largest problem being the difficulty of preventing unwanted levels of change in the specimen through the extended exposure to a hostile environment of X-rays and, in some cases, high vacuum and/or extreme cold. The present paper discusses possible methods of dealing with this problem.

\section{Background}

In the single-particle technique, a small non-crystalline particle is placed in a coherent monochromatic X-ray beam and its diffraction pattern is collected, phased and Fourier inverted to give the specimen image. The image-forming procedure is thus very similar to that which, in X-ray crystallography, is done with a crystalline specimen. The singleparticle diffraction pattern however does not show the gathering of the diffraction pattern into the strong Bragg-spot pattern which is characteristic of the crystal case, and this makes necessary a much increased exposure of the specimen in the X-ray beam and, thus, for radiation-sensitive specimens, increased specimen change and correspondingly lower imaging resolution. For the biological cell, the 2005/06 work leads to an estimate, when techniques have been further optimized, of perhaps $10 \mathrm{~nm}$ imaging resolution for the twodimensional imaging of the cell. That resolution, if maintained in three-dimensional imaging, could offer cell biologists new methods of studying important aspects of cell behavior, such as how the cell spatially deploys its large molecular assemblies in handling the situations which it is called upon to deal with.

\section{Current work}

In our cell preparation, we start with the belief that the best first step is to plunge-freeze the living cell, allowing it to be captured, in its current instantaneous state, in whatever conditions the cell biologist wishes to have it, and with minimal introduction of foreign matter of any kind. At this point, the cell is in the frozen-hydrated condition, and the water in the cell is in the form of vitreous ice. The frozenhydrated cell is, in some respects, a very favorable object for high-quality imaging: the structure is closest to the living structure; it is physically bolstered by the solid ice; and the structure-damaging free radicals produced by exposure to photons are largely caught and immobilized in the ice. There are, however, serious negatives as well: except in the water window (the photon energy region from 284 to $540 \mathrm{eV}$ in which water is much more transparent than protein), cell contrast is weak; in the water window, cells larger than 3-4 $\mu \mathrm{m}$ are too opaque for good imaging; also even small changes in the character and/or distribution of the specimen's highly labile ice component can compromise the accurate measurement of the weak speckle (non-Bragg spot) diffraction pattern. We thus foresee certain speciality uses for the frozenhydrated cell, but think that frozen-dehydrated cells, usable at ordinary temperatures, will be the more widely used ones. The above-mentioned 2005/06 work made use of the simplest type of frozen-dehydrated cells, in which the only processing of the frozen-hydrated cell was that of slow careful in vacuo warming and drying. Such cells, if not exposed to X-rays, can in many cases start living again when they are restored to an aqueous environment.

A recent StonyBrook/Berkeley/Cornell two-dimensional imaging of a yeast cell is shown in Fig. 1. As noted, the 2005/06 work was two-dimensional in nature; thus nearly all collection of the diffraction pattern was with the cell always in the same orientation in the X-ray beam. This had the effect of making the cell appear to be less damaged in the beam than was really 
the case. During the work, however, as attention started to be turned toward three-dimensional imaging and the cell began to be more extensively rotated in the beam, it became clear that the photon beam does distort the frozen-dehydrated specimen, mainly by compressing it along the beam direction; the yeast cell, at least in the simplest frozen-dehydrated state, is not strong enough to remain truly unchanged in the beam for the length of time needed to collect the three-dimensional data set. Thus possible variations in the dehydration process should now be studied in more detail, as well as various types of chemical processing to give further strength to the structure and/or label particular components of the structure. My personal feeling is that, if biologists start to take up the singleparticle technique, such possibilities will be found. We are, after all, only dealing at present with quite low resolution (10 nm) imaging.

\section{Stereoscopic viewing}

In addition to the above possibilities, however, there is the possibility of lowering the amount of X-ray exposure needed to convert from two-dimensional to three-dimensional imaging. The Hegerl-Hoppe theorem on dose fractionation appears to lend some basis for optimism in this direction. Our plan is to explore this, beginning with the simplest elaboration over the two-dimensional imaging that we can think of, namely the step from diffraction with the specimen in one orientation in the X-ray beam to diffraction with it in two orientations, i.e. to the change from monoscopic to stereoscopic viewing. Two-orientation viewing is known to give a very considerable degree of three-dimensional perception, and at the same time it only increases beamtime exposure over two-dimensional imaging by a factor of two instead of by the

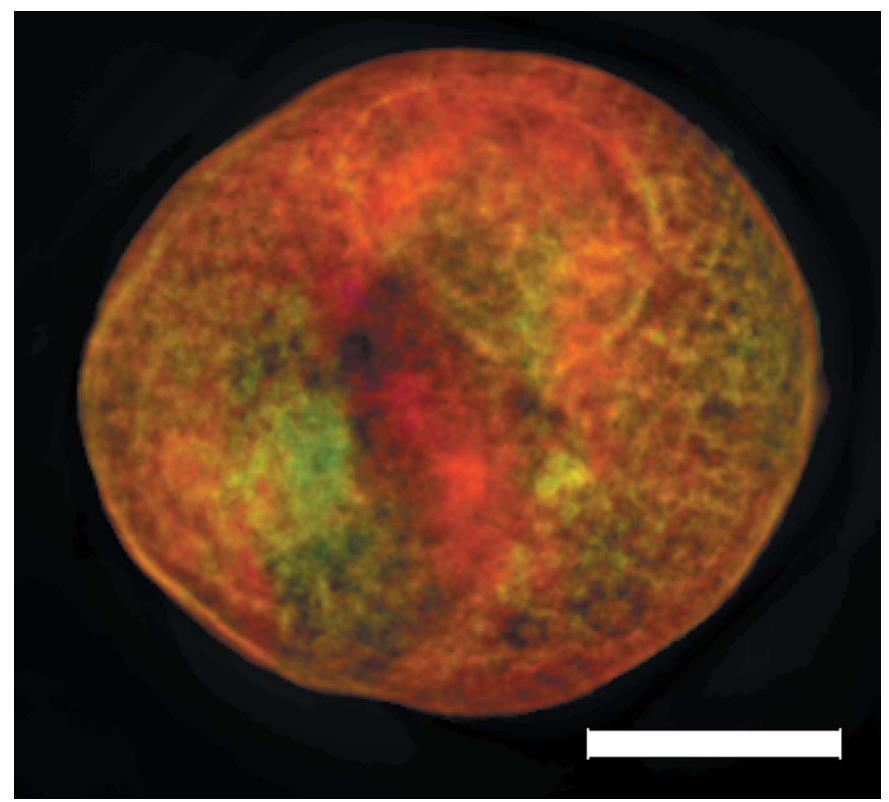

Figure 1

Two-dimensional image of a yeast cell (scale bar $1 \mathrm{um}$ ). Hue represents the phase and brightness the amplitude of the X-ray exit wave. several hundred required for full multi-orientational viewing. Thus, using the term stereo-three-dimensional for this type of three-dimensional viewing, it should be possible on a synchrotron source to get a single frozen-hydrated or frozendehydrated cell to last long enough in the beam for several differently oriented stereo-three-dimensional viewings at $10 \mathrm{~nm}$ resolution. Or, given a FEL flash X-ray source employing beam splitting to hit the specimen with the two viewing-angle beams simultaneously, an individual cell can undergo only a single stereo-three-dimensional viewing; here, however, it is the X-ray pulse shape which largely takes over command of resolution, and that stereo-three-dimensional imaging may reach near $1 \mathrm{~nm}$ resolution. Also, given the flash $\mathrm{X}$-ray source, no need for online cryogenics exists; the X-ray flash itself should equal or surpass the thermal flash as a means of capturing the exact structure of the wet and living cell or, if the greater contrast of the frozen-dehydrated cell is desired, that cell can be prepared offline and simply introduced into the flash apparatus for stereo-three-dimensional viewing.

\section{Summary}

On the question of whether cells are sturdy enough to let us see them in three dimensions via X-ray diffractive imaging, Mother Nature has not yet told us her answer. Success in the use of the frozen-hydrated cell appears to require scrupulous attention to detail in cryogenic diffraction technique, and will still leave us with a rather low contrast type of specimen. Still, because of its strong protectiveness of the original structure, this may be the best overall solution to the puzzle. Use of the less strongly protected frozen-dehydrated cell currently appears to be blocked by the cell-compression effect of the photon beam, but a number of cell-strengthening techniques, having also considerable labeling and information-adding benefits, remain to be tried. We plan also to try stereoscopic viewing, with its lower beam exposure, and think that it is the dehydrated cell, through the expected openness of its structure, which may be best suited to that type of viewing.

It is appropriate for this Special Issue that some very early acknowledgements on this work go back exactly to 1948, for it was in that year that A. L. Patterson, Henry Lipson and Raymond Pepinsky suggested that I choose the Fourier transform in X-ray structure analysis as a Master's Degree thesis topic; the fact that it was on the transform, not the series, meant that my mind would be opened to the possibility of the non-crystalline single particle becoming a valid type of specimen for X-ray diffraction. Also in the next year, when I was a doctoral student in England, I met Alan Turing, and he made me aware of Shannon's theorem, which in turn enabled me to see the great phasing benefit that would result if we could sample the transform more finely than is done by the Bragg spots when the specimen is a crystal. Now, 60 years later, these diverse concepts have at last joined together into a whole. All the persons whom I have mentioned are gone, but it should be known that their actions have lived on. Present-day work on single-particle diffraction imaging, and on closely 
related methods, is now in the hands of many excellent researchers [for an outline of what is taking place, see the accompanying addendum kindly contributed by David Shapiro (2008)]; and this note in particular, on the special problem of imaging the biological cell, could not have been written without the contributions made through the years by the members of the StonyBrook/Berkeley/Cornell team, all of whom have played a part in the work. My thanks go particularly to Janos Kirz, now at Berkeley, Chris Jacobsen at Stony Brook, Veit Elser and Pierre Thibault at Cornell, David Shapiro now at Berkeley, Andrew Stewart at Stony Brook, Enju Lima at ESRF, and Huijie Miao, Xiaojing Huang, Jan Steinbrener and Johanna Nelson at Stony Brook; I bear the responsibility for this assessment of the current situation, however.
This project is supported by the National Institutes of Health, the National Science Foundation and Department of Energy grants to the Stony Brook group. The Advanced Light Source at Lawrence Berkeley National Laboratory is supported by the Director, Office of Science, Office of Basic Energy Sciences, Materials Sciences Division, of the US Department of Energy.

\section{References}

Shapiro, D. (2008). Acta Cryst. A64, 36-37.

Shapiro, D., Thibault, P., Beetz, T., Elser, V., Howells, M., Jacobsen, C., Kirz, J., Lima, E., Miao, H., Neiman, A. \& Sayre, D. (2005). Proc. Natl Acad. Sci. USA, 102, 15343-15346.

Thibault, P., Elser, V., Jacobsen, C., Shapiro, D. \& Sayre, D. (2006). Acta Cryst. A62, 248-261. 